Pacific Journal of Mathematics

MATRIX REPRESENTATIONS FOR LINEAR
TRANSFORMATIONS ON SERIES ANALYTIC IN THE UNIT
DISC

PHILIP C. TONNE 


\title{
MATRIX REPRESENTATIONS FOR LINEAR TRANSFORMATIONS ON SERIES ANALYTIC IN THE UNIT DISC
}

\author{
Philip C. TONNE
}

Let $S$ be the space of all complex sequences $A$ such that if $z$ is a complex number and $|z|<1$ then $\sum A_{n} z^{n}$ converges. We present three characterizations of the linear transformations from $S$ to $S$ which have matrix representations. We also characterize the linear transformations from $S$ to the bounded sequences (or to the convergent sequences) which have matrix representations. The characterizations are in terms of natural topologies for the spaces.

These results are a blend of Köthe and Toeplitz' much quoted study [3] of complex sequence spaces, Haplanov's beautiful characterization [1] of those matrices which transform $S$ to $S$, and some rather natural norms for $S$ which have been used by V. Ganapathy Iyer [2] in his study of entire functions.

Köthe and Toeplitz study complex sequence spaces, matrices, and linear transformations having a kind of continuity which is independent of norms. A space is said to be normal provided that if $x$ is in the space and $\left|y_{n}\right| \leqq\left|x_{n}\right|, n=0,1, \cdots$, then $y$ is also in the space. Köthe and Toeplitz show that a continuous linear transformation from a normal space to a normal space has a matrix representation, and conversely, provided that each space contains all the "finite" sequences. Our space $S$ is normal and the space of bounded sequences is also normal. The continuity criteria used in our theorems (statement (2) in each) are special cases of the continuity condition of Köthe and Toeplitz. It follows from their work that the existence of a matrix for a linear transformation $L$ is necessary and sufficient for $L$ to have "analytic" continuity (see definition below).

Given a matrix transformation from $S$ to $S$ and a norm $N_{r}(0<r<1$ : if $A$ is in $\left.S, N_{r}(A)=\sum_{p=0}^{\infty}\left|A_{p}\right| r^{p}\right)$ for $S$, Haplanov's theorem provides another such norm $N_{R}$ such that the transformation is continuous from the normed linear space $\left\{S, N_{r}\right\}$ to $\left\{S, N_{R}\right\}$. Finally, to complete Theorem 1, each linear transformation which is continuous relative to some such pair of norms is represented by a matrix, even though $S$ is complete with respect to neither of the norms.

Our second theorem is like the first: the transformations are from $S$ to the bounded sequences (or convergent sequences).

In [6] Wilanski gives a result of a similar kind for convergencepreserving transformations. 
For a topological approach to obtaining continuity for a transformation from the existence of a matrix representation, see Wilanski's Functional Analysis [5, p. 204].

The author is indebted to the referee for most of the references.

THEOREM 1. Suppose that $L$ is a linear transformation from $S$ to $S$. These statements are equivalent:

(1) $L$ has a matrix representation (there is a complex matrix $M$ such that if $A$ is in $S$ and $n$ is a nonnegative integer then $L(A)_{n}=$ $\left.\sum_{k=0}^{\infty} M_{n k} A_{k}\right)$.

(2) If $A$ is a sequence of sequences in $S$ and $A$ has limit 0 analytically (definition below), then the sequence $\left\{L\left(A_{n}\right)\right\}_{n=0}^{\infty}$ has limit 0 analytically also.

(3) If $0<R<1$ then there is a number $r$ between 0 and 1 such that $L$ is a continuous transformation from the normed linear space $\left\{S, N_{r}\right\}$ to $\left\{S, N_{R}\right\}$. (For $\rho$ between 0 and 1 and $A$ in $S, N_{\rho}(A)=$ $\sum_{n=0}^{\infty}\left|A_{n}\right| \rho^{n}$.)

(4) There are numbers $r$ and $R$ between 0 and 1 such that $L$ is a continuous linear transformation from $\left\{S, N_{r}\right\}$ to $\left\{S, N_{R}\right\}$.

Definition. If $A$ is a sequence of sequences in $S$ and $f$ is a sequence of analytic functions such that if $n$ is a nonnegative integer and $|z|<1$ then

$$
f_{n}(z)=\sum_{k=0}^{\infty} A_{n k} z^{k}
$$

and $f$ converges uniformly with limit 0 on each closed subset of the unit disc, then $A$ is said to have limit 0 analytically.

HAPLANOV's THEOREM. Suppose that $M$ is an infinite complex matrix. Then these statements are equivalent:

(1) If $A$ is in $S$ then $M \cdot A$ is in $S$. $\left((M \cdot A)_{n}=\sum_{k=0}^{\infty} M_{n k} A_{k}\right.$.)

(2) There are numbers $r$ and $d$ such that $0<r<1$ and $d$ is $a$ positive integer such that if $j$ and $k$ are nonnegative integers and $k>j d+d$ then $\left|M_{j_{k}}\right|<r^{k}$, and there is a sequence $s$ in $S$ such that $\left|M_{j k}\right| \leqq s_{j}(j, k=0,1, \cdots)$.

THEOREM 2. Suppose that $L$ is a linear transformation from $S$ to the bounded sequences (convergent sequences). Then these statements are equivalent:

(1) L has a matrix representation.

(2) If $A$ is a sequence with values in $S$ and has limit 0 analytically, then $\left\{L\left(A_{n}\right)\right\}_{n=0}^{\infty}$ is a sequence with limit 0 in the least upper bound norm.

(3) There is a number $r$ between 0 and 1 such that if $0<R<1$ 
then $L$ is continuous from $\left\{S, N_{r}\right\}$ to $\left\{S, N_{R}\right\}$.

(4) There is a number $r$ between 0 and 1 such that $L$ is a continuous transformation from $\left\{S, N_{r}\right\}$ to the space of bounded sequences (convergent sequences) under the least upper bound norm.

The matrix transformations from $S$ to the bounded sequences (convergent sequences) are characterized as follows [4]:

Theorem A. Suppose that $M$ is an infinite complex matrix. Then these statements are equivalent:

(1) If $A$ is in $S$, then $M \cdot A$ is a bounded (convergent) sequence.

(2) Each column of $M$ is a bounded (convergent) sequence and there are numbers $r$ and $t$ such that $0<r<1$ and if each of $j$ and $k$ is a nonnegative integer then $\left|M_{j k}\right| \leqq t r^{k}$.

The following notation and lemmas are useful in the proofs of our theorems.

Notation. $l_{1}$ is the space of all sequences $x$ such that $\sum\left|x_{k}\right|$ converges, and $N_{1}$ is its usual norm: if $x$ is in $l_{1}$ then $N_{1}(x)=$ $\sum_{k=0}^{\infty}\left|x_{k}\right|$. If each of $x$ and $y$ is a sequence, then $x \cdot y$ is the sequence such that if $n$ is a nonnegative integer then $(x \cdot y)_{n}=x_{n} \cdot y_{n}$.

Cauchy's Inequalities. Suppose that $A$ is in $S, 0<r<1$, and $\mu$ is a number such that if $|z|=r$ then $\left|\sum_{n=0}^{\infty} A_{n} z^{n}\right| \leqq \mu$. Then, for each nonnegative integer $n,\left|A_{n}\right| r^{n} \leqq \mu$.

LEMma 0. If $A$ is a sequence of sequences in $S$ which has limit 0 analytically and $B$ is the sequence such that

$$
B(n)_{k}=B_{n k}=\max \left\{\left|A_{n j}\right|, j=0,1, \cdots, k\right\}(n, k=0,1, \cdots),
$$

then $B$ has limit 0 analytically.

Proof. Let $r$ be a number between 0 and 1 . Let $R$ be a number between $r$ and 1. Let $\varepsilon$ be a positive number. Let $m$ be a positive integer such that if $n$ is an integer exceeding $m$ and $|z| \leqq R$ then $\left|\sum_{k=0}^{\infty} A_{n k} z^{k}\right|<\varepsilon /(1-r / R)$. Let $n$ be an integer exceeding $m$. By Cauchy's inequalities, $\left|A_{n k}\right|<\varepsilon R^{-k} /(1-r / R), k=0,1, \cdots$. For each nonnegative integer $k$ let $j_{k}$ be a nonnegative integer such that $j_{k} \leqq$ $k$ and $B_{n k}=\left|A\left(n, j_{k}\right)\right|$. If $k$ is a nonnegative integer, $R^{-k} \geqq R^{-j_{k}}$. Consequently, 


$$
\begin{gathered}
\sum_{k=0}^{\infty} B_{n k} r^{k}=\sum_{k=0}^{\infty}\left|A\left(n, j_{k}\right)\right| r^{k} \\
<\sum_{k=0}^{\infty} \frac{\varepsilon}{1-r / R} R^{-k} r^{k}=\varepsilon .
\end{gathered}
$$

Lemma 1. Suppose that $A$ is a sequence (of sequences in $S$ ), $A$ has limit 0 analytically, and $C$ is the sequence such that if each of $n$ and $k$ is a nonnegative integer then $C(n)_{k}=C_{n k}=\left|A_{n k}\right|$. Then $C$ has limit 0 analytically.

Proof. This follows immediately from Lemma 0.

Lemma 2. Suppose that $A$ is a sequence, $A$ has limit 0 analytically, and $s$ is in $S$. Let $C$ be the sequence such that if each of $n$ and $k$ is a nonnegative integer then $C(n)_{k}=C_{n k}=A_{n k} s_{k}$. Then $C$ has limit 0 analytically.

Proof. Let $r$ be a number between 0 and 1 and let $R$ be a number between $r$ and 1. Recall the Cauchy-Hadamard characterization for $S:$ the sequence $x$ belongs to $S$ only in the case that lim $\sup _{n}\left|x_{n}\right|^{1 / n} \leqq$ 1. Therefore there is a number $t$ such that if $k$ is a nonnegative integer then $\left|s_{k} r^{k}\right|<t R^{k}$. Then, if $n$ is a nonnegative integer and $|z| \leqq r$,

$$
\begin{aligned}
\left|\sum_{k=0}^{\infty} C_{n k} z^{k}\right| & \leqq \sum_{k=0}^{\infty}\left|A_{n k} s_{k}\right| r^{k} \\
& \leqq t \sum_{k=0}^{\infty}\left|A_{n k}\right| R^{k},
\end{aligned}
$$

so that by Lemma $1, C$ has limit 0 analytically.

LEMma 3. If $A$ is a sequence which has limit 0 analytically, $d$ is a positive integer, and $C$ is the sequence such that if each of $n$ and $k$ is a nonnegative integer then $C(n)_{k}=C_{n k}=A(n, k d+d)$, then $C$ has limit 0 analytically. Furthermore, if $0<r<1$ and $R=r^{1 / d}$ then $N_{r}\left(C_{n}\right) \leqq N_{R}\left(A_{n}\right) / r, n=0,1, \cdots$.

Proof. Let $r$ be a number between 0 and 1 . Let $R$ be $r^{1 / d}$ and let $\varepsilon$ be a positive number. By Lemma 1 there is is a positive integer $m$ such that if $n$ is an integer exceeding $m$ then $\sum_{k=0}^{\infty}\left|A_{n k}\right| R^{k}<r \cdot \varepsilon$. Let $n$ be an integer exceeding $m$. Then, if $|z| \leqq r$,

$$
\begin{aligned}
\left|\sum_{k=0}^{\infty} C_{n k} z^{k}\right| & \leqq N_{r}\left(C_{n}\right)=\sum_{k=0}^{\infty}|A(n, k d+d)| r^{k} \\
& =\sum_{k=0}^{\infty}|A(n, k d+d)| R^{-d} R^{k d+d} \leqq R^{-d} \sum_{k=0}^{\infty}\left|A_{n k}\right| R^{k}<\varepsilon,
\end{aligned}
$$


and

$$
N_{r}\left(C_{n}\right) \leqq R^{-d} N_{R}\left(A_{n}\right)=\frac{1}{r} N_{R}\left(A_{n}\right) .
$$

LEMmA 4. Suppose that $0<a<1, R=a^{2}, s$ is in $S$, and $s_{k} \geqq 0, k=0,1, \cdots$ Then $s^{2}$ in $S$ and $N_{R}\left(s^{2}\right) \leqq N_{a}(s)^{2}$.

Proof.

$$
\begin{aligned}
N_{R}\left(s^{2}\right) & =N_{a^{2}}\left(s^{2}\right)=\sum_{n=0}^{\infty} s_{n}^{2}\left(a^{2}\right)^{n}=\sum_{n=0}^{\infty} s_{2 n-n} s_{n} a^{2 n} \\
& \leqq \sum_{n=0}^{\infty}\left(\sum_{k=0}^{2 n} s_{2 n-k} s_{k}\right) a^{2 n} \leqq \sum_{n=0}^{\infty}\left(\sum_{k=0}^{n} s_{n-k} s_{k}\right) a^{n} \\
& =\left(\sum_{n=0}^{\infty} s_{n} a^{n}\right)^{2}=N_{a}(s)^{2} .
\end{aligned}
$$

Lemma 5. Suppose that $0<r<1$ and $A$ is in $S$ and $B$ is the sequence such that if $n$ is a nonnegative integer then $B_{n}=$ $\max \left\{\left|A_{k}\right|, k=0, \cdots, n\right\}$. Then

$$
N_{r}(B) \leqq N_{r}(A) /(1-r) \text {. }
$$

Proof.

$$
\begin{aligned}
N_{r}(B) & =\sum_{n=0}^{\infty} B_{n} r^{n} \leqq \sum_{n=0}^{\infty}\left(\sum_{k=0}^{n}\left|A_{k}\right|\right) r^{n} \\
& =\sum_{k=0}^{\infty}\left|A_{k}\right| \sum_{n=k}^{\infty} r^{n}=\sum_{k=0}^{\infty}\left|A_{k}\right| r^{k} \sum_{n=0}^{\infty} r^{n} \\
& =N_{r}(A) /(1-r) .
\end{aligned}
$$

Proof of Theorem 1. (1 $\rightarrow 2)$ Statement (2) follows statement (1) as a consequence of Köthe and Toeplitz' Satz 2, $\S 8$ [3, p. 208]. Lemma 1 (above) is useful in showing that our "analytic" continuity is equivalent to their continuity. Alternately, one can cite a general theorem concerning $F K$ spaces [5, Cor. 5, p. 204], or else one can use Haplanov's Theorem and Lemmas $0,1,2$, and 3.

$(2 \rightarrow 1)$ Statement (1) follows from (2) as a consequence of Satz $7, \S 6[3$, p. 207]. This can also be done by modifying the argument under the heading $(4 \rightarrow 1)$ below.

$(1 \rightarrow 3)$ Let $R$ be a number between 0 and 1 , and let $M$ be the matrix representation for $L$. Let $s$ be the sequence such that if $n$ is a nonnegative integer then $s_{n}=\sum_{k=0}^{\infty}\left|M_{n k}\right|$. By Haplanov's Theorem, $s$ is in $S$, and there are numbers $q$ and $d$ such that $0<q<1$ and $d$ 
is a positive integer such that if $j$ and $k$ are nonnegative integers and $k>j d+d$ then $\left|M_{j k}\right|<q^{k}$. Let $r$ be a number between $q$ and 1 and between $R^{1 / 2 d}$ and 1 .

Let $A$ be in $S$ and suppose that each of $B$ and $C$ is a sequence such that

$$
B_{n}=\max \left\{\left|A_{j}\right|, j=0,1, \cdots n\right\} \quad \text { and } \quad C_{n}=B_{n d+d}(n=0,1, \cdots) .
$$

Each of $B$ and $C$ is in $S$, and, by Cauchy's inequality and Lemmas 4, 3, and 5 ,

$$
\begin{aligned}
N_{R}(L(A)) & =\sum_{n=0}^{\infty}\left|\sum_{k=0}^{\infty} M_{n k} A_{k}\right| R^{n} \\
& \leqq \sum_{n=0}^{\infty} \sum_{k=0}^{n d+d}\left|M_{n k}\right|\left|A_{k}\right| R^{n}+\sum_{n=0}^{\infty} \sum_{k=n d+d+1}^{\infty}\left|M_{n k}\right|\left|A_{k}\right| R^{n} \\
& \leqq \sum_{n=0}^{\infty} s_{n} B_{n d+d} R^{n}+\sum_{n=0}^{\infty} \sum_{k=0}^{\infty} q^{k}\left|A_{k}\right| R^{n} \\
& \leqq \sum_{n=0}^{\infty} s_{n} R^{n / 2} C_{n} R^{n / 2}+\frac{1}{1-R} \sum_{k=0}^{\infty}\left|A_{k}\right| q^{k} \\
& \leqq\left(\sum_{n=0}^{\infty} s_{n}^{2} R^{n}\right)^{1 / 2}\left(\sum_{n=0}^{\infty} C_{n}^{2} R^{n}\right)^{1 / 2}+N_{q}(A) /(1-R) \\
& \leqq N_{\sqrt{\bar{R}}}(s) N_{\sqrt{\bar{R}}}(C)+N_{q}(A) /(1-R) \\
& \leqq R^{-1 / 2} N_{\sqrt{\bar{R}}}(s) N_{r}(B)+N_{r}(A) /(1-R) \\
& \leqq \frac{R^{-1 / 2}}{1-r} N_{\sqrt{\bar{R}}}(s) N_{r}(A)+\frac{1}{1-R} N_{r}(A)
\end{aligned}
$$

$L$ is a continuous linear transformation from $\left\{S, N_{r}\right\}$ to $\left\{S, N_{R}\right\}$.

$(4 \rightarrow 1)$ For each nonnegative-integer pair $\{j, k\}$ let $M_{j_{k}}$ be $L\left(\delta_{k}\right)_{j}$. $\left(\left(\delta_{k}\right)_{j}=1\right.$ if $j=k,\left(\delta_{k}\right)_{j}=0$ otherwise.) Let $B$ be a member of $S$ and let $n$ be a nonnegative integer. Recall that $l_{1}$ is a subset of the ring $S$ and let $T$ be that linear transformation from $l_{1}$ to the complex plane such that if $A$ is in $l_{1}$ then $T(A)=L(B \cdot A)_{n}$. If $A$ is a sequence of sequences in $l_{1}$ and the sequence $\left\{N_{1}\left(A_{j}\right)\right\}_{j=0}^{\infty}$ has limit 0 , then $\left\{N_{r}\left(B \cdot A_{j}\right)\right\}_{j=0}^{\infty}$ has limit 0 , so that $\left\{N_{R}\left(L\left(B \cdot A_{j}\right)\right)\right\}_{j=0}^{\infty}$ has limit 0 and $\left\{T\left(A_{j}\right)\right\}_{j=0}^{\infty}$ has limit 0 (by Cauchy's inequalities). $T$ is a continuous linear transformation from $l_{1}$ to the plane. Consequently, there is a bounded complex sequence $b$ such that if $x$ is in $l_{1}$ then $T(x)=\sum_{k=0}^{\infty} b_{k} x_{k}$. Furthermore, if $k$ is a nonnegative integer then

$$
b_{k}=T\left(\delta_{k}\right)=L\left(B \cdot \delta_{k}\right)_{n}=L\left(B_{k} \cdot \delta_{k}\right)_{n}=B_{k} \cdot L\left(\delta_{k}\right)_{n}=M_{n k} B_{k} \cdot
$$

Let $A$ be the sequence whose only value is $1 . A$ is in $S$. Let $x$ be the sequence with values in $l_{1}$ such that if each of $j$ and $k$ is a nonnegative integer then $x_{j k}=1$ if $j \geqq k$ and $x_{j k}=0$ if $j<k$. The 
sequence $\left\{N_{r}\left(B \cdot\left(x_{j}-A\right)\right)\right\}_{j=0}^{\infty}$ has limit 0 . So $\left\{N_{R}\left(L\left(B \cdot\left(x_{j}-A\right)\right)\right)\right\}_{j=0}^{\infty}$ has limit 0 , and, by Cauchy's inequalities, $\left\{L\left(B \cdot x_{j}\right)_{n}\right\}_{j=0}^{\infty}$ has limit $L(B \cdot A)_{n}=L(B)_{n}$ : the sequence $\left\{T\left(x_{j}\right)\right\}_{j=0}^{\infty}$ has limit $L(B)_{n}$. But, if $j$ is a nonnegative integer,

$$
T\left(x_{j}\right)=\sum_{k=0}^{\infty} b_{k} x_{j k}=\sum_{k=0}^{j} b_{k}=\sum_{k=0}^{j} M_{n k} B_{k},
$$

and

$$
L(B)_{n}=\sum_{k=0}^{\infty} M_{n k} B_{k}
$$

so $L$ has a matrix representation.

Statement (4) follows immediately from (3). So we have proved Theorem 1.

Proof of Theorem 2. $(1 \rightarrow 2)$ One can again quote [3, Satz 2, p. 208] or quote [5, Cor. 5, p. 204] or simply apply Theorem A and Lemma 1.

$(1 \rightarrow 4)$ Let $M$ be the infinite matrix such that if $x$ is a bounded (convergent) sequence then $L(x)_{n}=\sum_{k=0}^{\infty} M_{n k} x_{k}(n=0,1, \cdots)$. There are numbers $r$ and $t$ such that $\left|M_{j k}\right| \leqq t r^{k}(j, k=0,1, \cdots)$. Let $A$ be in $S$ and let $j$ be a nonnegative integer.

$$
\left|L(A)_{j}\right|=\left|\sum_{k=0}^{\infty} M_{j k} A_{k}\right| \leqq t \sum_{k=0}^{\infty} r^{k}\left|A_{k}\right|=t N_{r}(A) .
$$

Hence, $L$ is a continuous transformation from $\left\{S, N_{r}\right\}$ to the space of bounded sequences (convergent sequences) with least upper bound norm.

$(2 \rightarrow 1)$ Suppose that $A$ is a sequence having limit 0 analytically and $0<r<1$. Let $\varepsilon$ be a positive number and let $m$ be a positive integer such that if $n$ is an integer exceeding $m$ then

$$
\left|L\left(A_{n}\right)_{k}\right|<\varepsilon(1-r) \quad(k=0,1, \cdots) .
$$

Then, if $|z| \leqq r$ and $n$ is an integer exceeding $m$,

$$
\left|\sum_{k=0}^{\infty} L\left(A_{n}\right)_{k} z^{k}\right| \leqq \sum_{k=0}^{\infty}\left|L\left(A_{n}\right)_{k}\right| r^{k}<\varepsilon .
$$

Consequently, the sequence $\left\{L\left(A_{n}\right)\right\}_{n=0}^{\infty}$ has limit 0 analytically, so that by Theorem $1, L$ has a matrix representation.

$(4 \rightarrow 3)$ Let $r$ be a number between 0 and 1 such that $L$ is a continuous transformation from $\left\{S, N_{r}\right\}$ to the space of bounded sequences (convergent sequences) under the least upper bound norm. Suppose that $0<R<1$. 
There is a number $K$ such that if $A$ is in $S$ then

$$
\left|L(A)_{j}\right| \leqq K N_{r}(A) \quad(j=0,1, \cdots) .
$$

So, if $A$ is in $S$,

$$
N_{R}(L(A))=\sum_{j=0}^{\infty}\left|L(A)_{j}\right| R^{j} \leqq \frac{K}{1-R} N_{r}(A),
$$

and $L$ is continuous from $\left\{S, N_{r}\right\}$ to $\left\{S, N_{R}\right\}$.

Statement (1) follows from statement (3) by Theorem 1, and so we are finished with the proof of Theorem 2.

\section{REFERENCES}

1. M. G. Haplanov, Linear transformations of analytic spaces, Amer. Math. Soc. Transl., (2) 13 (1960), 177-183.

2. V. Ganapathy Iyer, On the spaces of integral functions, Proc. Amer. Math. Soc., 3 (1952), 874-883.

3. G. Köthe and $\mathrm{O}$. Toeplitz, Lineare Räume mit unendlichvielen Koordinaten und Ringe unenlicher Matrizen, Journal für die reine und angewandte Mathematik, 171 (1934), 193-226.

4. P. C. Tonne, Matrix transformtions on the power series convergent on the unit disc,

J. London, Math. Soc., (2), 4 (1972), 667-670.

5. Albert Wilanski, Functional Analysis, Blasisdell, New York, 1964.

6. - Subalgebras of $B(X)$, Proc. Amer. Math. Soc., 29 (1971), 355-360.

Received July 23, 1971

EMORY UNIVERSITY 


\section{PACIFIC JOURNAL OF MATHEMATICS}

\section{EDITORS}

H. SAMELSON

Stanford University

Stanford, California 94305

C. R. Hовву

University of Washington

Seattle, Washington 98105
J. DugundJI

Department of Mathematics University of Southern California

Los Angeles, California 90007

RICHARD ARENS

University of California

Los Angeles, California 90024

\section{ASSOCIATE EDITORS}

E. F. BECKENBACH

B. H. NeumanN

F. WOLF

K. YoSHIDA

\section{SUPPORTING INSTITUTIONS}

UNIVERSITY OF BRITISH COLUMBIA

CALIFORNIA INSTITUTE OF TECHNOLOGY

UNIVERSITY OF CALIFORNIA

MONTANA STATE UNIVERSITY

UNIVERSITY OF NEVADA

NEW MEXICO STATE UNIVERSITY

OREGON STATE UNIVERSITY

UNIVERSITY OF OREGON

OSAKA UNIVERSITY
UNIVERSITY OF SOUTHERN CALIFORNIA

STANFORD UNIVERSITY

UNIVERSITY OF TOKYO

UNIVERSITY OF UTAH

WASHINGTON STATE UNIVERSITY

UNIVERSITY OF WASHINGTON

AMERICAN MATHEMATICAL SOCIETY

NAVAL WEAPONS CENTER

Printed in Japan by International Academic Printing Co., Ltd., Tokyo, Japan 


\section{Pacific Journal of Mathematics}

\section{Vol. 44, No. $1 \quad$ May, 1973}

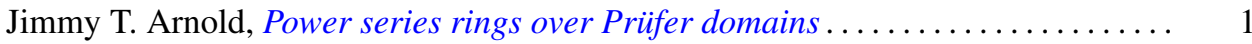

Maynard G. Arsove, On the behavior of Pincherle basis functions . . . . . . . . . 13

Jan William Auer, Fiber integration in smooth bundles ................. 33

George Bachman, Edward Beckenstein and Lawrence Narici, Function algebras

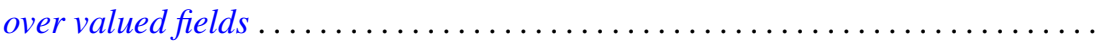

Gerald A. Beer, The index of convexity and the visibility function . . . . . . . . . . .

James Robert Boone, A note on mesocompact and sequentially mesocompact

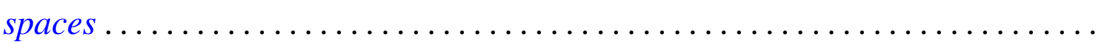

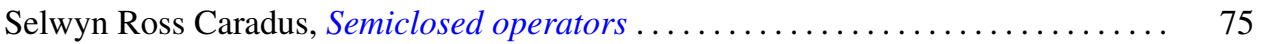

John H. E. Cohn, Two primary factor inequalities . . . . . . . . . . . . . . . 81

Mani Gagrat and Somashekhar Amrith Naimpally, Proximity approach to

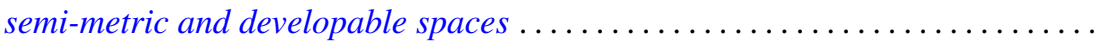

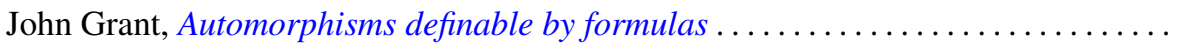

Walter Kurt Hayman, Differential inequalities and local valency ..............

Wolfgang H. Heil, Testing 3-manifolds for projective planes . . . . . . . . . . . . .

107

Melvin Hochster and Louis Jackson Ratliff, Jr., Five theorems on Macaulay

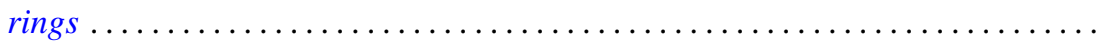

Thomas Benton Hoover, Operator algebras with reducing invariant subspaces ....

James Edgar Keesling, Topological groups whose underlying spaces are separable

Fréchet manifolds...

Frank Leroy Knowles, Idempotents in the boundary of a Lie group . .

191

George Edward Lang, The evaluation map and EHP sequences ...

201

Everette Lee May, Jr, Localizing the spectrum . . . . . . . . . . . .

211

Frank Belsley Miles, Existence of special $K$-sets in certain locally compact abelian groups.

Susan Montgomery, A generalization of a theorem of Jacobson. II . .

T. S. Motzkin and J. L. Walsh, Equilibrium of inverse-distance forces in

three-dimensions.

Arunava Mukherjea and Nicolas A. Tserpes, Invariant measures and the converse

of Haar's theorem on semitopological semigroups .

James Waring Noonan, On close-to-convex functions of order $\beta$

Donald Steven Passman, The Jacobian of a growth transformation

Dean Blackburn Priest, A mean Stieltjes type integral ........ .

Joe Bill Rhodes, Decomposition of semilattices with applications to topological

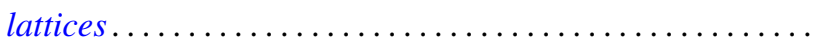

Claus M. Ringel, Socle conditions for $\mathrm{QF}-1$ rings ..........

Richard Rochberg, Linear maps of the disk algebra

Roy W. Ryden, Groups of arithmetic functions under Dirichlet convolution . .

Michael J. Sharpe, A class of operators on excessive functions

Erling Stormer, Automorphisms and equivalence in von Neumann algebras ..

Philip C. Tonne, Matrix representations for linear transformations on series

analytic in the unit disc. 